\title{
BARE STRANGE QUARK STARS: FORMATION AND EMISSION
}

\author{
Renxin Xu \\ School of Physics, Peking University, Beijing 100871, China; rxxu@bac .pku . edu .cn
}

A theory is just a mathematical model, that describes and codifies the observations.

- A man having a belief in Positivist Philosophy

\begin{abstract}
Recent achievements of bare strange stars are briefly reviewed. A nascent protostrange star should be bare because of strong mass ejection and high temperature after the supernova detonation flame, and a crust can also hardly form except for a super-Eddington accretion. The magnetosphere of a bare strange star is composed mainly of $e^{ \pm}$pair plasma, where both inner and outer vacuum gaps work for radio as well as high energy nonthermal emission. A featureless thermal spectrum is expected since no ion is above the quark surface, whilst electron cyclotron lines could appear in some bare strange stars with suitable magnetic fields. Various astrophysical implications of bare strange stars are discussed.
\end{abstract}

Keywords: pulsars, neutron stars, dense matter, elementary particles

\section{Introduction}

Identifying strange quark stars (or simply strange stars, SS) is among the most important problems in modern astrophysics. Strange stars (SSs) are hypothetical compact objects that consists of roughly equal numbers of deconfined up, down and strange quarks, to affirm or negate the existence of which should have profound implications in the study of the elemental strong interaction (see, e.g., Xu 2002a, for a review). 
It becomes more and more recognized in astrophysical community, that those compact objects, previously thought as neutron stars (NSs), may actually be SSs. Whatever the objects are, the most essential and important thing is to find clear observational signatures of SSs or NSs.

Bare strange stars (BSSs) are SSs with bare quark surfaces, i.e., without possible crusts of ordinary matter. Recently, much attention is paid to the study of BSSs, because the peculiarity of quark surface properties may eventually help us to identify a quark star (e.g., Xu 2002a). Although most of literatures are concerned with crusted strange stars (e.g., Madsen 1999, for a review), several groups are doing care about the study of the physics and astrophysics of BSSs. Usov (2002, for a review) proposed a mechanism for $e^{ \pm}$(and thus photon) emission in the superstrong electric field above the surface of a hot BSS, and considered possible heating evens of BSSs in order to explain some astronomical phenomena; while Ray et al. (2000) calculated the mass-radius relations of BSSs with finite temperatures (upto $70 \mathrm{MeV}$ ). Due to the abrupt density change, from $\sim 4 \times 10^{14} \mathrm{~g} / \mathrm{cm}^{3}$ to 0 in $\sim 1 \mathrm{fm}$, BSSs may collapse into black holes via microscopic black holes seeding (Gorham et al. 2002). Motivated by the nonconservation of the quark axial current at MIT bag boundary, Ng, Cheng \& Chu (2002) proposed a pion cloud, with a thick of $\sim 1 \mathrm{fm}$, around a BSS, which may affect significantly the cooling curves of BSS. However, if SSs appear as radio pulsars, plenty of data of which are accumulated, what can the observation tell us about the nature of pulsars? It is necessary to combine the researches of radio pulsars, NSs, and SSs (see, Xu, Zhang \& Qiao 2001a, for a review) all together in order to have an effective study.

We will focus the review, in this paper, on the formation and emission of BSSs, with the inclusion of various astrophysical implications of BSSs.

\section{Formation of bare strange stars}

A protostrange star should be bare. No numerical model of supernova explosion known hitherto has included the conversion from protoneutron stars to protostrange stars although such a conversion may be helpful in modelling the burst process. Nevertheless, some efforts have been made in trying to understand the transition and combustion processes in detail. From a kinetic point of view, Olinto (1987) for the first time calculated the conversion of neutron stars into strange stars, suggesting a deflagration mode with a burning velocity range from $10^{4} \mathrm{~km} / \mathrm{s}$ to a few $\mathrm{cm} / \mathrm{s}$. However it is found (Horvath \& Benvenuto 1988; Benvenuto, Horvath \& Vucetich 1989; Benvenuto \& Horvath 1989) that such slow modes are unstable. This instability would be self-accelerated, and the 
burning should occur finally in detonation modes although the transition from deflagration to detonation has not been well understood (Lugones, Benvenuto \& Vucetich 1994). It is almost impossible that a crust can survive the detonation flame. First, the total released phase transition energy $\sim 10^{52} M_{1}$ ergs is much greater than the crust gravitational binding energy $\sim 10^{48} M_{1}^{2} / R_{6}$ ergs, where $M_{1}$ is the SS mass in $M_{\odot}, R_{6}$ the radius in $10 \mathrm{~km}$. Second, the rate of energy release when forming a strange star, $\sim 10^{53} \mathrm{ergs} / \mathrm{s}$, is much larger than the Eddington luminosity, $\sim 10^{38} \mathrm{ergs} / \mathrm{s}$. In addition, high temperature $(\sim 30 \mathrm{MeV})$ of newborn BSSs increases the quantum penetration rate of ions (Usov 1998), and the electric field can only thus sustain a crust with mass $\ll 10^{-5} M_{\odot}$.

Can a nascent BSS be covered via accretion in its future? It was believed that, during and after supernova explosion, material with ejection speed smaller than that of escape should fall back to the center star with hypercritical accretion rate (Chevalier 1989). However whether this initial super-Eddington accretion occurs is still a matter of debate because of the untractable nature of modelling supernova explosion with the inclusion of rapid rotation and strong magnetic field. Owing to rapid rotation and strong magnetic fields, most of the fallback matter may form temporarily a fossil disk, and the accretion onto stellar surface can only be impossible when the rotation period $P$ satisfies (Xu et al 2001a),

$$
P>3.2 \times 10^{3} B_{12}^{6 / 7} R_{6}^{18 / 7} M_{1}^{-5 / 7} \dot{M}_{10}^{-3 / 7} \mathrm{~s},
$$

where $B=10^{12} B_{12} \mathrm{G}$ is the magnetic field, and $\dot{M}=10^{10} \dot{M}_{10} \mathrm{~g} / \mathrm{s}$ is the accretion rate. Also, a $3-\mathrm{D}$ magnetohydrodynamic simulations, not including stellar rotation and B-fields, showed that the gravitational energy of the infall-magnetized plasma has to be converted to other energies and that the initial accretion rate migth be reduced significantly (Igumenshchev \& Narayan 2002). Furthermore, as argued by Xu (2002b), a crust can not form by accretion as long as the accretion rate $<\mathcal{L}^{*}$,

$$
\mathcal{L}^{*} \sim \frac{9.1 \times 10^{35}}{\varepsilon} M_{1} R_{6}^{-1} P^{-1 / 2} \operatorname{ergs~s}^{-1},
$$

with $5 \times 10^{-5} / P<\varepsilon<0.5$. In conclusion, BSSs can exist in nature, some of which may be active as radio pulsars, anomalous X-ray pulsars, soft $\gamma$-ray repeators, etc. (Table 1 ).

\section{Magnetospheric and thermal emission}

BSS emission can be divided into magnetospheric and thermal parts.

One of the consequences of strong binding of charged particles (quarks and electrons) on BSS surfaces is the formation of an RS-type (Ruderman \& Sutherland 1975) vacuum inner gap above polar cap (Xu \& Qiao 
1998, Xu, Qiao \& Zhang 1999). Although radio pulsar emission mechanism is not well understood, the RS model is still the most popular one to connect magnetospheric dynamics with general observations, with a "user friendly" nature. The RS model may naturally explain many observational features of radio pulsars, such as drifting subpulses (e.g., Deshpande \& Rankin 1999), microstructures (Hankins 1996), and even the core-cone beams (Qiao \& Lin 1998) and their polarizations (Xu et al. 2000a). However, if radio pulsars are NSs with canonical dipole magnetic fields, the RS model faces at least two difficulties: the binding energy problem and the antipulsar issue. These two can easily be overcome if BSSs are chosen as the nature of radio pulsars.

Nonetheless the RS model may still work, but requires special conditions, for neutron stars (Gil \& Mitra 2001, Gil, Melikidze \& Mitra 2001, Gil \& Melikidze 2002). Since ion cohesive energy is higher for a stronger magnetic field, the binding energy crisis for NSs could be solved if a complicated multipolar magnetic field, with a field strength being much higher than that of the dipole component and a curvature radius being much smaller than the stellar one, is assumed. Whereas there are at least two points to be questioned in this scenario: 1, the RS model can not work for antipulsars; 2, the cohesive energy in reality could be much smaller than that of condensed iron matter since the bombardment of backflow particles, with an energy $>\mathrm{GeV}$ in the frame of center of mass, may convert heavy elements into lighter ones by nuclear reactions $(\mathrm{Xu}$ et al. 2000b). Besides, the field configuration is also a mater of debate.

In addition, the existence of vacuum outer gap can also reflect the strong binding of particles on BSS surface. A plasma magnetosphere, with charge density of $\rho_{\text {GJ }}$ (Goldreich \& Julian 1969) to quench $E_{\|}$(the surface electric fields being parallel to the magnetic fields) induced by the unipolar effect, should surround a BSSs; any charge departure from $\rho_{\text {GJ }}$ has to result in acceleration, the region of which is called "gap". Owing to the force-free flow along spiral magnetic field lines near light cylinder (Fig.4a in Holloway 1975) and the centrifugal force, the charge density in the open-field-line region can not be $\rho_{\mathrm{GJ}}$; gaps form then in this region. Whereas the open-field-line region can in principal be divided into two regions: region I with polar radius $r_{\mathrm{p}+}^{\mathrm{d}}$ and region II between field lines "a" and "b" (see Fig.1 of Ruderman \& Sutherland 1975); the charge signs of these two are opposite outside the light cylinder. A natural way to close the electric current in BSS magnetospheres is that an RS-type inner vacuum gap forms above the polar cap in region I while an outer one (e.g., Cheng, Ho \& Ruderman 1986) develops near the null surface in region II. However there may be some unseemly points for the outer gap if pulsars are not BSSs but NSs. 1. Because the field lines are 
equipotential, for a parallel rotator as an example, electrons can freely flow from stellar surface in all the open region; particles of the opposite sign may emitted in a "thin sheath" (Michel 1975). 2. Since negatively charged particles flow out in region I, positively charged ions in region II may flow freely from stellar surface through the null surface if the binding energy of ions is not high enough (Holloway 1975). In both cases the outer vacuum gap can not work for NSs.

Actually observational supports (e.g., Romani \& Yadigaroglu 1995; Wang, Xu \& Qiao 2002) could be hints of the existence of outer gaps, and thus of the strong binding of charges on BSS surface. Certainly it is very necessary to improve gap models of BSSs, especially for the interaction between inner and outer vacuum gaps; a dynamically consistent model with both kinds of gaps may be essential to understand the observations. It is worth noting that the null surface is not necessary to serve as the inner boundary of the outer gap; it may be possible that the inner boundary of the P-N junction (Holloway 1973) could be much lower (Hirotani \& Shibata 2001).

Let's turn to the discussion of BSS thermal emission. In principle, one can study the thermally radiative properties by comparison of theoretically modelled spectra with that of observations, in order to get a real information of photons from quark matter astrophysically (whereas direct photons and lepton pairs have been recognized to be the clearest signatures for quark-gluon plasma in terrestrial physics, e.g., Cassing \& Bratkovskaya 1999). Unfortunately no emergent spectrum of BSSs appears in literature although some efforts were tried (Chmaj et al. 1991, Page \& Usov 2002, Ng et al. 2002). Nevertheless, as argued by Xu (2002b), the cooling and the thermal radiation of a BSS may not be strongly conflict with observations when polar cap heating is considered. The total luminosity of BSS thermal emission, including photons and $e^{ \pm}$pairs, was calculated (see Usov 2001a for the details) in the frame of Usov (1998).

$\mathrm{Xu}$ (2002b) suggested that a featureless thermal spectrum could be a probe for identifying strange stars, since no bound charged particle is in discrete quantum states on the quark surface without strong magnetic field; but discrete Landau levels appear for charged particles in strong fields, which could result in a cyclotron line spectrum. In fact it is a central goal and a real competition among the observers to find line emission in the thermal radiation of NS atmospheres, since the stellar mass $M$ and radius $R$ may be derived by obtaining its gravitational redshift (as $M / R$ ) and the pressure broadening (as $M / R^{2}$ ) of the lines. More advanced facilities, Chandra and XMM-Newton, make this inves- 
tigation possible. Still almost no line is observed (Table 1) except for those sources 1E 1207.4-5209, SGR 1806-20, and EXO 0748-676.

Table 1. "NSs" with thermal X-ray spectrum observed by Chandra, XMM, or others

\begin{tabular}{|c|c|c|c|}
\hline Name & Period (s) & B-field (G) & Age (yr) \\
\hline RX J1856.5-3754 (INS $\left.{ }^{a}\right)$ & $\ldots$ & & $\ldots$ \\
\hline RX J0720.4-3125 (INS) & 8.39 & $\sim 10^{13}$ & $\sim 10^{6}$ \\
\hline 1E 1048.1-5937 $\left(\mathrm{AXP}^{b}\right)$ & 6.45 & Magnetar? & $\ldots$ \\
\hline $4 \mathrm{U} 0142+61(\mathrm{AXP})$ & 8.69 & Magnetar? & $\ldots$ \\
\hline PSR J0437-4715 (msPSR $\left.{ }^{c}\right)$ & 0.00576 & $3 \times 10^{8}$ & $4.9 \times 10^{6} ?$ \\
\hline PSR B0833-45 (Vela) & 0.0893 & $3.4 \times 10^{12}$ & $1.1 \times 10^{4}$ \\
\hline PSR B0656+14 & 0.385 & $4.7 \times 10^{12}$ & $1.0 \times 10^{5}$ \\
\hline Kes $79\left(\mathrm{CCO}^{d}\right)$ & 8.39 & $\ldots$ & $0.6-1.2 \times 10^{4} ?$ \\
\hline Cas A (CCO) & $0.0122 ?$ & $<5 \times 10^{10} ?$ & \\
\hline Pup A (CCO, RXJ0822) & $\ldots$ & $\ldots$ & $\sim 4 \times 10^{3}$ \\
\hline RX J1308.6+2127 (INS) & 5.16 & $1.7-3.2 \times 10^{14} ?$ & $\ldots$ \\
\hline RX J0806.4-4123 (INS) & 11.37 & $\sim 5 \times 10^{10} ?$ & $\ldots$ \\
\hline 1E 1207.4-5209 (CCO, PKS1209) & 0.424 & $\sim 10^{11} ?$ & $(3-20) \times 10^{3} ?$ \\
\hline SGR 1806-20 $\left(\mathrm{SGR}^{e}\right)$ & 7.47 & $\sim 5 \times 10^{11} ?$ & $\ldots$ \\
\hline EXO 0748-676 (X-ray burster) & $\ldots$ & $\ldots$ & $\ldots$ \\
\hline
\end{tabular}

References - Zane et al. (2002), Seward et al. (2002), Murray et al. (2001), Zavlin et al. (1999), Kaplan et al. (2002), Haberl \& Zavlin (2002), Hambaryan et al. (2002), Sanwal et al. (2002), Xu et al. (2003), Ibrahim et al. (2002), Cottam et al. (2002), and those references related to Table 1 of $\mathrm{Xu}(2002 \mathrm{~b})$.

${ }^{a}$ INS: isolated neutron stars. ${ }^{b} \mathrm{AXP}$ : anomalous X-ray pulsars. ${ }^{c} \mathrm{PSR}$ : pulsars, msPSR: millisecond pulsars. ${ }^{d} \mathrm{CCO}$ : compact central objects in SNRs. ${ }^{e}$ SGR: soft $\gamma$-ray repeaters.

Sanwal et al. (2002) supposed a likely interpretation in which the absorption features in 1E 1207.4-5209 are associated with atomic transitions, and that it is very difficult to interpret the absorption features in term of cyclotron lines. However this opinion was criticized by $\mathrm{Xu}$ et al. (2003), who proposed that 1E 1207.4-5209 may have a debris disk and is in a propeller phase, with an accretion rate $\sim 6 \times 10^{-11} M_{\odot} /$ year. Ibrahim et al. (2002) detected features in the bursts of a soft-gammarepeater SGR 1806-20, which is supposed as the proton cyclotron lines in superstrong magnetic field $\left(\sim 10^{15} \mathrm{G}\right)$. Whilst, due to the high massenergy $(\sim 1 \mathrm{GeV})$ of a proton, the ratio of the oscillator strength of the first harmonic to that of fundamental in $10^{15} \mathrm{G}$ is only $\sim 10^{-6}$ ! It is not reasonable to detect the first and the even higher harmonics. In fact, numerical spectrum simulations of atmospheres with protons in superstrong fields have never show more than two proton absorption lines (Ho \& Lai 2001). Motivated by these, Xu et al. (2003) suggested that the observed features could be electron cyclotron lines, and that 
SGR 1806-20 may have an ordinary magnetic field, $\sim 5 \times 10^{11}$ G. Also RX J0806.4-4123 shows a possible absorption at $0.4-0.5 \mathrm{keV}$ (Haberl \& Zavlin 2002), which corresponds a B-field of $\sim 5 \times 10^{10} \mathrm{G}$ if being cyclotron-originated. Why only these in which significant absorption features have been detected so far? Xu et al. (2003)'s answer is that, for detectors with observing energy from $\sim 0.1$ to $\sim 10 \mathrm{keV}$, the sensitive magnetic fields in which electrons can absorb resonantly photons within that energy range are from $9 \times 10^{9} \mathrm{G}$ to $1 \times 10^{12} \mathrm{G}$. No objects with certain magnetic field strength, listed in the upper part of Table 1, shows significate absorption feature. It is worth noting that magnetospheric power law components of BSSs are also featureless (Xu \& Qiao 1998, Xu et al. 2001a), but a neutron star may have magnetospheric line features because of the ions, pulled out from NS surface by the space-charge-limited-flow mechanism, in the open field line region.

Although BSSs may be common, SSs with their historical accretion rates being much higher than the Eddington one could be crusted. Xray bursters, if being SSs, might be in this case. Cottam et al. (2002) discovered significant absorption lines in the spectra of EXO 0748-676, all with a readshift 0.35 . This result can not rule out an SS model for EXO 0748-676 (Xu 2002c).

\section{Conclusions and Discussions}

The formation of BSSs is reasonable, and the BSS thermal and magnetospheric emission could be consistent with observations. It is a hot topic now to discuss the astrophysical implications of SSs (Dai \& Lu 1998, Cheng et al. 1998), whereas more attention should be paid to the bare quark surface in the future.

There may be some other implications of BSSs.

1. Strong magnetic fields of radio pulsars (and others) are essential to understand the observational data. This field could be influenced significantly by, e.g., Hall drift and Ohmic decay (Hollerbach \& Rüdiger 2002) in the crusts if pulsars are NSs or SSs with crusts, but may keep constantly for BSS with color superconductivity (Xu \& Busse 2001).

2. It is a strange and interesting thing that the masses of observed stellar black hole candidates are $>\sim 7 M_{\odot}$ whereas the mass limit of Fermion stars are $1-3 M_{\odot}$. Why do not we find black holes with $\sim$ $3-7 M_{\odot}$ ? This discrepancy may be explained if we assume that: a, massive main-sequence stars have very rapid rotating cores in the highly evolved phases; b, quark matter in strange stars can be enough confined against the centrifugal breaking. In this case, it is found that the masses of stellar black holes should be greater than $6 M_{\odot}$ if a black hole can 
only form when the stellar radius of a BSS is smaller than the radius of its minimum stable orbit (Zhang, Zhang \& Xu 2003).

3. The soft $\gamma$-ray bursts of SGR 0526-66, with peak luminosity $10^{7} L_{\text {Edd }}$, needs ultra-strong field $\left(\sim 10^{17} \mathrm{G}\right)$ to constrain the fireball. An alternative binding is through the quark surface; and it may be natural to explain the bursting energy and the light curves in a framework that a comet-like object falls to a BSS (Zhang et al. 2000, Usov 2001b).

4. It is suggested that the beam widths and polarizations of radio pulsars can be used to derive their mass-radius relations (Kapoor \& Shukre 2001), especially for the fastest rotating pulsar, PSR 1937+21 (Xu et al. 2001b), with the inclusion of general relativistic effects. Taking the simplest proposal that the inclination and impact angles are $90^{\circ}$ and $0^{\circ}$, respectively, $\mathrm{Xu}$ et al. (2001b) found stringent limits on the mass $M$ and the radius $R: M<0.2 M_{\odot}$ and $R<1 \mathrm{~km}$. If so, PSR $1937+21$ can not be an NS or an SS with a crust, but only a BSS, according to the mass-radius relations of SSs (e.g., Xu 2002c).

\section{Acknowledgments}

This work is supported by National Nature Sciences Foundation of China (10273001) and the Special Funds for Major State Basic Research Projects of China (G2000077602).

\section{References}

Benvenuto, O.G., Horvath, J.E. 1989, Phys. Rev. Lett., 63, 716

Benvenuto, O.G., Horvath, J.E., Vucetich, H. 1989, Int. J. Mod. Phys. A4, 257

Cassing, W., Bratkovskaya, E. L. 1999, Phys. Rep., 308, 65

Cheng, K. S., Dai, Z. G., Wei, D. M., Lu, T. 1998, Sci., 280, 407

Cheng, K. S., Ho, C., \& Ruderman, M. A. 1986, ApJ, 300, 500

Chevalier, R. A. 1989, ApJ, 346, 847

Cottam, J., Paerels, F., Mendez, M. 2002, Nature, 420, 51 (astro-ph/0211126)

Dai, Z. G., Lu, T. 1998, Phys. Rev. Lett., 81, 4301

Deshpande, A. A., Rankin, J. M. 1999, ApJ, 524, 1008

Gil, J. A., Melikidze, G. I. 2002, ApJ, 577, 909

Gil, J. A., Melikidze, G. I., Mitra, D. 2002, A\&A, 388, 246

Gil, J. A., Mitra, D. 2001, ApJ, 550, 383

Goldreich, P., \& Julian, W. H. 1969, ApJ, 157, 869

Gorham, P., Learned, J., Lehtinen, N. 2002 (astro-ph/0205170)

Haberl, F., Zavlin, V. E. 2002, A\&A, 391, 571

Hambaryan, V., Hasinger, G., Schwope, A. D., Schulz, N. S. 2002, A\&A, 381, 98

Hankins, T. H. 1996, in: Pulsars: problems and progress, ASPC Ser., Vol. 105; eds. S. Johnston, M.A. Walker, \& M. Bailes., 197

Hirotani, K., Shibata, S. 2001, ApJ, 558, 216

Ho, W. C. G., Lai, D. 2001, MNRAS, 327, 1081

Hollerbach, R., Rüdiger, G. 2002, MNRAS, 337, 216 
Holloway, N. J. 1973, Nat. Phys. Sci., 246, 6

Holloway, N. J. 1975, MNRAS, 171, 619

Horvath, J.E., Benvenuto, O.G. 1988, Phys. Lett., B213, 516

Ibrahim, A. I., et al. 2002, ApJ, 574, L51

Igumenshchev, I. V., Narayan, R. 2002, ApJ, 566, 137

Kaplan, D. K., Kulkarni, S. R., van Kerkwijk M. H. 2002, ApJL, in press

Kapoor, R. C., Shukre, C. S. 2001, A\&A, 375, 405

Lugones, G., Benvenuto, O.G., Vucetich, H. 1994, Phys. Rev. D50, 6100

Madsen, J. 1999, in Hadrons in Dense Matter and Hadrosynthesis (Springer), 162

Michel, F. C. 1975, ApJ, 197, 193

Murray, S. S. 2001, preprint (astro-ph/0106516)

Ng, C. Y., Cheng, K. S., Chu, M. C. 2002, Astropart. Phys., in press (astro-ph/0209016)

Olinto, A.V. 1987, Phys. Lett. B192, 71

Page, D., Usov, V. V. 2002, Phys. Rev. Lett., 89, 131101

Qiao, G. J., Lin, W. P. 1998, A\&A, 33, 172

Ray, S., Dey, J., Dey, M., Ray, K., Samanata, B. C. 2000, A\&A, 364, L89

Romani, R. W., Yadigaroglu, I. A. 1995, 1995, ApJ, 438, 314

Ruderman, M. A., \& Sutherland, P. G. 1975, ApJ, 196, 51

Sanwal, D., Pavlov, G. G., Zavlin, V. E., Teter, M. A. 2002, ApJ, 574, L61

Seward, F. D., Slane, P. O., Smith, R. K. 2002 (astro-ph/0210496)

Usov, V.V. 1998, Phys. Rev. Lett., 81, 4775

Usov, V. V. 2001a, ApJ, 550, L179

Usov, V. V. 2001b, Phys. Rev. Lett. 87, 021101

Usov, V. V. 2002, eConf C010815, 36 (astro-ph/0111442)

Wang, H.G., Xu, R.X., Qiao, G.J. 2002, ApJ, 578, 385

Xu, R.X. 2002a, in High Energy Processes, Phenomena in Astrophysics, Proceedings of IAU Symposium No. 214, eds. X. D. Li et al., in press (astro-ph/0211348)

Xu, R.X. 2002b, ApJ, 570, L65

Xu, R.X. 2002c, preprint (astro-ph/0211214)

Xu, R.X., Busse, F.H. 2001, A\&A, 371, 963

Xu, R.X., Liu, J.F., Han, J.L., Qiao, G.J., 2000a, ApJ, 535, 354

Xu, R. X., \& Qiao, G. J. 1998, Chin. Phys. Lett., 15, 934

Xu, R. X., Qiao, G. J., \& Zhang, B. 1999, ApJ, 522, L109

Xu, R. X., Qiao, G. J., \& Zhang, B. 2000b, in: Pulsar Astronomy - 2000 and beyond, ASPC Ser. Vol. 202, eds. M. Kramer, N. Wex \& R. Wielebinski, 479

Xu, R. X., Wang, H. G., Qiao, G. J. 2003, Chin. Phys. Lett. in press (astro-ph/0207079)

Xu, R.X., Xu, X.B., Wu, X.J. 2001b, Chin. Phys. Lett., 18, 837 (astro-ph/0101013)

Xu, R. X., Zhang, B., Qiao, G. J. 2001a, Astropart. Phys., 15, 101

Zane, S., et al. 2002 (astro-ph/0203105)

Zavlin, V. E., Trümper, J., Pavlov, G. G. 1999, ApJ, 525, 959

Zhang, B., Xu, R. X, Qiao, G. J. 2000, ApJ, 545, L127

Zhang, W. M., Zhang, S. N., Xu, R. X. 2003, this proceedings. 